\title{
Coma science: intensive care as the new frontier
}

\author{
Jan Claassen * (1)
}

(0) 2019 Springer-Verlag GmbH Germany, part of Springer Nature

Beginning in the middle of the last century, increasingly large numbers of comatose patients have survived life-threatening conditions thanks to the widespread use of mechanical ventilation. The chances of reasonable functional outcomes for survivors of acute brain injury rose over the following years, largely due to advances and standardization of critical care management and improvements in neurosurgical techniques. Neurocritical care physicians routinely manage patients with acute brain injury, who are frequently unconscious. This young specialty has spent the past couple of decades making large strides in harmonizing clinical practice, advancing societal representation, and developing a unique, large body of evidence for critical care physicians treating patients with neurological injury. Having established national and international societies that represent practitioners of neurocritical care, generating guidelines and practice statements, and establishing organized training with board certification, the field is now undergoing a scientific transformation. At this junction, an increasing number of research groups are focusing attention on understanding disorders of consciousness in acutely brain-injured patients. Techniques for this coma science endeavor are now available to provide fundamental insights into the biology of consciousness of the acutely injured brain.

These efforts have become of utmost clinical and ethical significance given the progress in general critical care. Medical advances have created a scenario, where the majority of comatose patients with acute brain injury die from withdrawal of life support rather than cardiopulmonary collapse. The decision to withdraw life support

\footnotetext{
*Correspondence: jc1439@cumc.columbia.edu

Department of Neurology, Neurological Institute, New York Presbyterian

Hospital, Columbia University, 177 Fort Washington Avenue, MHB 8

Center, Room 300, New York, NY 10032, USA
}

is made collaboratively with health-care proxies, often the closest family, and the physicians taking care of the patient. However, our ability to predict outcomes, including recovery of consciousness in patients with traumatic brain injury or other acute brain insults such as stroke or cardiac arrest, is poor [1]. Subsequently, it is not surprising that there is significant variation in the rates of withdrawal of care in comparable patients [2]. This uncertainty leads itself to the self-fulfilling prophecy of a poor outcome. With this in mind, it becomes imperative to better understand the mechanisms underlying coma and develop more accurate prognosticators and even treatments for coma. While arguably being one of the central goals for research in the neurocritical care sphere, this work is also becoming increasingly achievable. Coma science in critical care builds on a rich foundation of insights gained from studying loss of consciousness due to sleep, anesthesia, and chronic disorders of consciousness [3]. This work has resulted in mechanistic models that provide a framework to conceptualize, study and potentially treat impairments of consciousness in critical care [1]. We are now beginning to understand the dynamic, complex interactions between brain regions that are required for the conscious state [4].

Clinical examinations have been the gold standard to assess consciousness, but these assessments are poorly standardized in critical care. The Coma Recovery Scalerevised [5], developed on a cohort of patients undergoing brain injury rehabilitation, is the industry standard to quantify consciousness in a scientific context (Fig. 1a). This scale allows categorization of patients into prognostically relevant groups of coma, vegetative state (also known as unresponsive wakefulness syndrome), minimally conscious state without (minus) or with (plus) inconsistent command following, and consciousness. However, obtaining the Coma Recovery Scale-revised may be time consuming, creating challenges in a critical

\section{Springer}




\section{A}

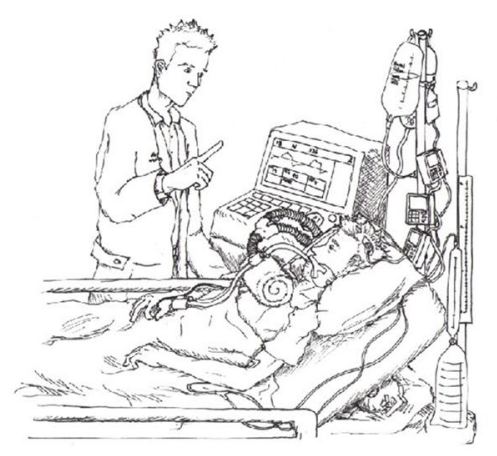

C

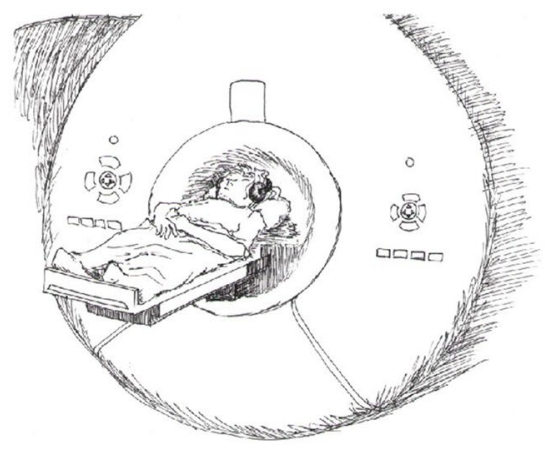

B

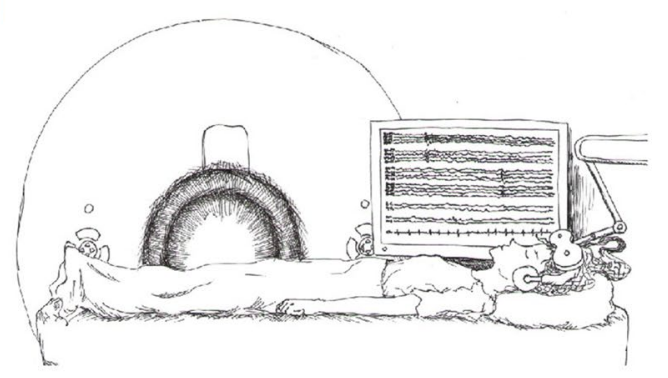

D

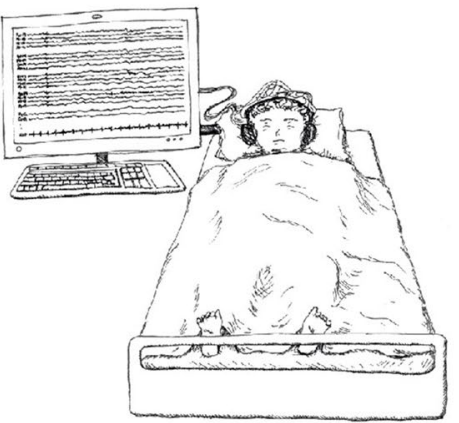

Fig. 1 a Health-care provider administering the Coma Recovery Scale-revised assessment. b Assessment of functional integration of information within thalamocortical circuits following MRI-guided targeted stimulation of brain regions using transcranial magnetic stimulation. c Functional MRI scan with auditory commands presented to a patient in the MRI scanner via headphones. $\mathbf{d}$ EEG assessment testing auditory command following using headphones

Illustrations by Dr Vivian Q Yang

care setting. More importantly, static behavioral assessments alone face major practical challenges early after brain injury, as the examination often fluctuates due to delirium, neurological improvement or worsening, medication or metabolic effects, and seizures. Consciousness determination by behavioral scales typically relies on comprehension of the command and detection of a motor response. Unfortunately, either may be impaired in brain-injured patients irrespective of any impairment of consciousness. More sensitive measures of responsiveness such as differential electromyographic recordings are promising and may be more sensitive and objective than relying on visual inspection by the examiner [6].

Strategies beyond behavioral assessments can be broadly conceptualized around assessments of the brain at rest, when confronted with a stimulus, or interventional studies (Table 1). These techniques each have their advantages and disadvantages which need to be carefully considered when used in the dynamic, stimulus-filled, critical care environment. Some diagnostic tests such as MRI and EEG may already be collected as part of routine clinical care, and systematic post-processing of this existing data can yield more objective correlates with consciousness and improve current prognostic schemes [7, 8]. Advanced neuroimaging assessments may add to future paradigms. Diffusion tensor imaging as an example quantifies structural connectivity, which may be particularly beneficial in prognosticating diffuse brain injuries such as that following cardiac arrest [9]. Functionally connected brain regions may also be unmasked using resting state MRI [10] or coherence analysis of the EEG recorded at rest [8]. Currently used serum, plasma, cerebrospinal fluid, and brain interstitial fluid measures of the extent of brain injury do not measure consciousness, but may have a role in prognostication. 
Table 1 Approaches to study consciousness in the acute brain injury setting

Brain at rest
Structural MR imaging (e.g., FLAIR, high-resolution T1, diffusion tensor imaging) [7, 9], head CT
Resting EEG, resting state functional MRI [8, 10]
Measures of the extent of brain injury in the serum, plasma, cerebrospinal fluid, and brain interstitial fluid (NSE, S1 10Beta, GFAP, vimentin, myelin basic
protein, inflammatory markers such as IgG electrophoresis)

Passive perturbation tasks

Long latency evoked potentials, event-related potentials [11, 12]

Autonomic nervous system modulation [13]

Transcranial magnetic stimulation with EEG co-registration [14] (Fig. 1b)

\section{Active perturbation tasks}

Behavioral assessment: Coma Recovery Scale-revised [5], other less appropriate clinical scales (FOUR score, Glasgow Coma Score) (Fig. 1a)

Differential electromyographic response [6]

Functional MRI—-motor imagery or activation paradigm [16, 17] (Fig. 1C)

Functional EEG—-motor imagery or activation paradigm [15, 17] (Fig. 1d)

Interventional studies (measures of success may include any of the above)

Medication (i.e., amantadine) [20]

Mechanical: electrical thalamic stimulation, focused ultrasound [19, 21]

References are just a small sample of available studies. More comprehensive reviews of the literature are available [1]. Citations [7-10, 12] were conducted in critical care setting

A significant body of literature has explored the brain's response to sensory stimuli in behaviorally unresponsive patients. Early adoptions included the development of mismatch negativity, contingent negative variation, and studying later components of evoked related potentials (i.e., P300 and N400). The ability to distinguish multiple layers of regularities in a stimulus may be particularly promising, as this may represent an auditory discrimination task and possibly a specific marker of conscious processing [11]. These event-related potentials can be obtained at the bedside in the critical care setting and relate to outcomes [12]. The impact of event-related cognitive processing on measures of the autonomic nervous system, such as heart rate variability, may provide a novel measure of residual cognitive processing [13]. This quantification of brain-body cross talk is uniquely attractive for the critical care setting, as continuous vital sign monitoring is abundantly available. In highly controlled settings, functional integration of information within thalamocortical circuits can be quantified with the perturbational complexity index (Fig. 1b) [14]. This measure is derived from high-density EEG recordings in response to stimulation of predefined brain regions using transcranial magnetic stimulation. However, the technical challenges in this approach prohibit its application in the critical care setting at this time.

Exploring brain responses to commands (active perturbation tasks), specifically in scenarios of asking behaviorally unresponsive patients to perform a motor command (i.e., "open and close your right hand"), has dramatically broadened our concepts of unresponsiveness. Up to
$15 \%$ of acutely brain-injured unresponsive patients had brain activation to motor commands and those with the response had a much higher chance of good functional outcome 1 year after the injury [15]. These findings have collectively established a state in which behavior and brain response are dissociated. Patients in this state, called cognitive motor dissociation, do not demonstrate any behavioral responses to motor commands, but functional MRI (Fig. 1c) or EEG (Fig. 1d) is able to detect brain activation that is strikingly similar to that of behaviorally responsive patients. First demonstrated in a chronically unresponsive patient following brain trauma [16], it has since been replicated in the critical care setting using both EEG and MRI $[15,17]$. It is unclear to what extent behaviorally unresponsive patients comprehend verbal commands, but the brain activation is uniquely different between the commands. Brain activation to a verbal motor command was found to be much more common in patients who had functional recovery 1 year after injury compared to patients who did not [15]. Combining imaging with EEG approaches is attractive as high-quality spatial and temporal information may be generated [17].

Therapeutic interventions with established targets have provided mechanistic insights into chronic disorders of consciousness and given hope for the development of treatments to directly support the recovery process [18]. Electrical stimulation of central thalamic nuclei [19] as well as dopaminergic activation with amantadine is linked to behavioral improvements in chronic disorders of consciousness [20]. Noninvasive, targeted stimulation 
using focused ultrasound may provide a therapeutic option, but also allow mechanistic investigations into the role of specific brain structures in disorders of consciousness [21]. Interventions will need to be measured not only by the impact on crude functional outcomes, but also include cognitive and quality of life measures.

The immediate, major challenge preventing scientific progress in this field is to overcome the therapeutic nihilism that unconscious brain-injured patients still face leading to the self-fulfilling prophecy of a poor outcome. Techniques that are low cost and easily applicable in the critical care setting are available and need to be further developed. Conceptually, bedside approaches are preferable early after the injury, as imaging techniques will require transport of critically ill patients which would be logistically challenging, expensive, and limited as it can not provide a dynamic, continuous assessment of a nonstatic patient examination early after brain injury. However, in the later subacute brain injury period, imaging modalities are promising to capture the extent and location of functional and structural disconnection in unresponsive patients.

Major collaborative efforts will be required to advance this field and meet the demands and needs of patients, families and physicians in this core area of critical care medicine. The international, multidisciplinary collaborative organized by the Neurocritical Care Society under the "Curing Coma" theme is a promising step in this direction. Efforts should be made to support and develop academic reward models that promote sharing of data and analytic code to promote collaborative research initiatives. Methodologic pitfalls should be openly discussed as the application of these techniques requires rigor in experimental design as well as computational analysis and interpretation of the data [22, 23]. Frameworks for data collection and analysis are being proposed and should be followed [24], and standardization and availability for interpretation require concerted efforts. Now is the time to advance the field of coma science which may yield more accurate assessments of the unresponsive acutely brain-injured patient, accurate prognostication of outcomes, and may even one day open communication channels to behaviorally unresponsive patients with cognitive motor dissociation [25]. Treatments to support recovery of consciousness may need to be individualized based on the specific location, extent and nature of the brain injury. This research has the potential to completely revolutionize management of acutely brain-injured patients, but at the same time may bring to surface ethical dilemmas that for a long time have been ignored. Accompanying the increasing availability of these techniques in clinical practice, a societal debate focusing on equity and societal burden needs to occur [1].
Acknowledgements

All images were prepared by Dr Vivian Q Yang.

Financial support and sponsorship

JC was supported by a grant funding from the NIH R01 NS106014 and R03 NS112760, and the DANA Foundation.

Compliance with ethical standards

\section{Conflicts of interest}

$J C$ is a minority shareholder at iCE Neurosystems. None of the authors have significant conflicts of interest to report.

\section{Publisher's Note}

Springer Nature remains neutral with regard to jurisdictional claims in published maps and institutional affiliations.

Received: 31 July 2019 Accepted: 9 October 2019

Published online: 20 November 2019

References

1. Posner JB, Saper CB, Schiff ND, Claassen J (2019) Plum and posners diagnosis and treatment of stupor and coma. Oxford University Press, Oxford

2. Turgeon AF, Lauzier F, Simard J-F et al (2011) Mortality associated with withdrawal of life-sustaining therapy for patients with severe traumatic brain injury: a Canadian multicentre cohort study. Can Med Assoc J 183:1581-1588. https://doi.org/10.1503/cmaj.101786

3. Giacino JT, Katz DI, Schiff ND et al (2018) Practice guideline update recommendations summary: disorders of consciousness: Report of the Guideline Development, Dissemination, and Implementation Subcommittee of the American Academy of Neurology; the American Congress of Rehabilitation Medicine; and the National Institute on Disability, Independent Living, and Rehabilitation Research. Neurology 91:450-460. https://doi.org/10.1212/WNL.0000000000005926

4. Demertzi A, Tagliazucchi E, Dehaene S, Deco G, Barttfeld P, Raimondo F, Martial C, Fernández-Espejo D, Rohaut B, Voss HU, Schiff ND, Owen AM, Laureys S, Naccache L, Sitt JD (2019) Human consciousness is supported by dynamic complex patterns of brain signal coordination. Sci Adv 5(2):eaat7603. https://doi.org/10.1126/sciadv.aat7603

5. Giacino JT, Kalmar K, Whyte J (2004) The JFK Coma Recovery ScaleRevised: measurement characteristics and diagnostic utility. Arch Phys Med Rehabil 85:2020-2029

6. Lesenfants D, Habbal D, Chatelle C et al (2016) Electromyographic decoding of response to command in disorders of consciousness. Neurology 87:2099-2107. https://doi.org/10.1212/WNL.0000000000003333

7. Rohaut B, Doyle KW, Reynolds AS et al (2019) Deep structural brain lesions associated with consciousness impairment early after hemorrhagic stroke. Sci Rep 9:4174. https://doi.org/10.1038/s41598-019-41042 $-2$

8. Claassen J, Velazquez A, Meyers E et al (2016) Bedside quantitative electroencephalography improves assessment of consciousness in comatose subarachnoid hemorrhage patients. Ann Neurol 80:541-553. https://doi. org/10.1002/ana.24752

9. Velly L, Perlbarg V, Boulier T et al (2018) Use of brain diffusion tensor imaging for the prediction of long-term neurological outcomes in patients after cardiac arrest: a multicentre, international, prospective, observational, cohort study. Lancet Neurol 17:317-326. https://doi.org/10.1016/ S1474-4422(18)30027-9

10. Threlkeld ZD, Bodien YG, Rosenthal ES et al (2018) Functional networks reemerge during recovery of consciousness after acute severe traumatic brain injury. Cortex 106:299-308. https://doi.org/10.1016/j.corte x.2018.05.004

11. Bekinschtein TA, Dehaene S, Rohaut B et al (2009) Neural signature of the conscious processing of auditory regularities. Proc Natl Acad Sci USA 106:1672-1677. https://doi.org/10.1073/pnas.0809667106 
12. Tzovara A, Rossetti AO, Juan E et al (2016) Prediction of awakening from hypothermic postanoxic coma based on auditory discrimination. Ann Neurol 79:748-757. https://doi.org/10.1002/ana.24622

13. Raimondo F, Rohaut B, Demertzi A et al (2017) Brain-heart interactions reveal consciousness in noncommunicating patients. Ann Neurol 82:578-591. https://doi.org/10.1002/ana.25045

14. Casali AG, Gosseries O, Rosanova M et al (2013) A theoretically based index of consciousness independent of sensory processing and behavior. Sci Transl Med 5:198ra105. https://doi.org/10.1126/scitransImed.3006294

15. Claassen J, Doyle K, Matory A et al (2019) Detection of brain activation in unresponsive patients with acute brain injury. N Engl J Med 380:24972505. https://doi.org/10.1056/NEJMoa1812757

16. Owen AM, Coleman MR, Boly M et al (2006) Detecting awareness in the vegetative state. Science 313:1402. https://doi.org/10.1126/science.11301 97

17. Edlow BL, Chatelle C, Spencer CA et al (2017) Early detection of consciousness in patients with acute severe traumatic brain injury. Brain 140:2399-2414. https://doi.org/10.1093/brain/awx176

18. Thibaut A, Schiff N, Giacino J et al (2019) Therapeutic interventions in patients with prolonged disorders of consciousness. Lancet Neurol 18:600-614. https://doi.org/10.1016/S1474-4422(19)30031-6

19. Schiff ND, Giacino JT, Kalmar K et al (2007) Behavioural improvements with thalamic stimulation after severe traumatic brain injury. Nature 448:600-603. https://doi.org/10.1038/nature06041
20. Giacino JT, Whyte J, Bagiella E et al (2012) Placebo-controlled trial of amantadine for severe traumatic brain injury. N Engl J Med 366:819-826. https://doi.org/10.1056/NEJMoa1102609

21. Monti MM, Schnakers C, Korb AS et al (2016) Non-invasive ultrasonic thalamic stimulation in disorders of consciousness after severe brain injury: a first-in-man report. Brain Stimul 9:940-941. https://doi.org/10.1016/j. brs.2016.07.008

22. Engemann DA, Raimondo F, King J-R et al (2018) Robust EEG-based crosssite and cross-protocol classification of states of consciousness. Brain 141:3179-3192. https://doi.org/10.1093/brain/awy251

23. Curley WH, Forgacs PB, Voss HU et al (2018) Characterization of EEG signals revealing covert cognition in the injured brain. Brain 141:1404-1421. https://doi.org/10.1093/brain/awy070

24. Comanducci A, Boly M, Claassen J, De Lucia M, Gibson RM, Juan E, Laureys S, Naccache L, Owen AM, Rosanova M, Rossetti AO, Schnakers C, Sitt J, Schiff ND The role of spontaneous and evoked EEG in the diagnostic and prognostic evaluation of acute and prolonged disorders of consciousness: recommendations for clinical practice and current research directions endorsed by the International Federation of Clinica. Clin Neurophysiol

25. Monti MM, Vanhaudenhuyse A, Coleman MR et al (2010) Willful modulation of brain activity in disorders of consciousness. N Engl J Med 362:579-589. https://doi.org/10.1056/NEJMoa0905370 